\title{
Fabrication of patterned calcium cross-linked alginate hydrogel films and coatings through reductive cation exchange
}

Marion Bruchet and Artem Melman*

Department of Chemistry and Biomolecular Science, Clarkson University

8 Clarkson Ave., Potsdam New York 13699-5810, USA

Phone +1 (315) 268-4405

Email: bruchem@clarkson.edu (Marion Bruchet)

amelman@clarkson.edu (Artem Melman)

\begin{abstract}
Calcium cross-linked alginate hydrogels are widely used in targeted drug delivery, tissue engineering, wound treatment, and other biomedical applications. We developed a method for preparing homogeneous alginate hydrogels cross-linked with $\mathrm{Ca}^{2+}$ cations using reductive cation exchange in homogeneous iron(III) cross-linked alginate hydrogels. Treatment of iron(III) cross-linked alginate hydrogels with calcium salts and sodium ascorbate results in reduction of iron(III) cations to iron(II) that are instantaneously replaced with $\mathrm{Ca}^{2+}$ cations, producing homogeneous ionically cross-linking hydrogels. Alternatively, the cation exchange can be performed by photochemical reduction in the presence of calcium chloride using a sacrificial photoreductant. This approach allows fabrication of patterned calcium alginate hydrogels through photochemical patterning of iron(III) cross-linked alginate hydrogel followed by the photochemical reductive exchange of iron cations to calcium.
\end{abstract}


Keywords: Alginate, Hydrogel, Ionic cross-linking, Iron, Photochemistry

\section{Introduction}

Alginates are natural anionic polysaccharide heteropolymers that consists of $(1,4)$ linked $\beta$-Dmannuronate $(\mathrm{M})$ and its $\mathrm{C} 5$-epimer, $\alpha$-L-guluronate $(\mathrm{G})$ residues, arranged in blocks of $\mathrm{M}-$, G-, and alternating G/M domains. Carboxylate groups of these residues are capable of cooperative binding with a variety of multivalent metal cations $\mathrm{M}^{\mathrm{n}+}$, resulting in formation of ionically cross-linked hydrogels. Calcium alginate is by far the most commonly ionically cross-linked hydrogel that is used in biomedical industry for wound treatment,(Boateng, Matthews, Stevens \& Eccleston, 2008; Lee \& Mooney, 2011) cell encapsulation,(Griffin \& Kasko, 2012; Tan \& Takeuchi, 2007) and as a scaffold for tissue engineering.(Kuo \& Ma, 2001; Shapiro \& Cohen, 1997) The dominant role in the cross-linking belongs to polyguluronate domains that bind $\mathrm{Ca}^{2+}$ cations in egg-box fashion(Grant, Morris, Rees, Smith \& Thom, 1973; Li, Fang, Vreeker \& Appelqvist, 2007; Sikorski, Mo, Skjak-Braek \& Stokke, 2007) According to the egg-box model (Fig. 1), faceting helical stretches of two polyguluronate domains provide chelate-type binding to $\mathrm{M}^{\mathrm{n}+}$ metal cations. Formation of these hydrogels takes place instantaneously upon mixing of solutions of alginate and metal cations such as preparation of alginate beads by dropwise addition of a solution of sodium alginate to a solution of metal cations. Hydrogels obtained by these methods are highly heterogeneous and their properties are difficult to control. Formation of homogeneous ionically cross-linked hydrogels can also be done by slow release of metal cations through exposure of a suspension of calcium carbonate particles in a solution of sodium alginate to gluconic acid produced through slow hydrolysis of gluconolactone(Kuo \& Ma, 2001), or released through UV irradiation of calcium complexes with photodegradable chelate ligands.(Chueh et al., 2010) However, these methods do not allows preparation of thin films and control of patterning of the resultant hydrogels. 


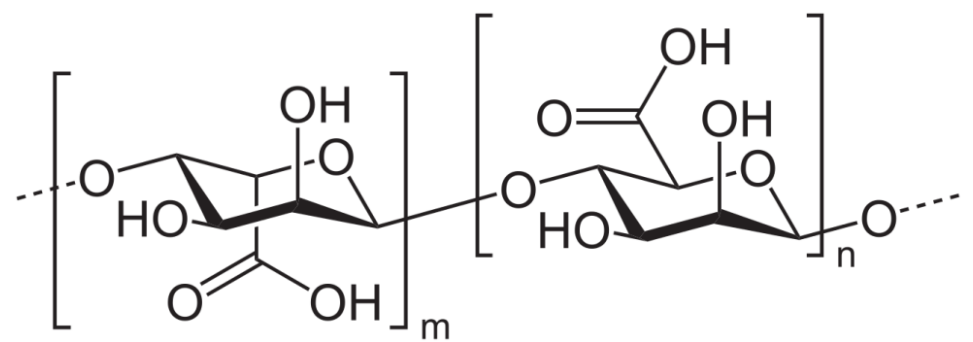

(G) guluronate

(M) mannuronate

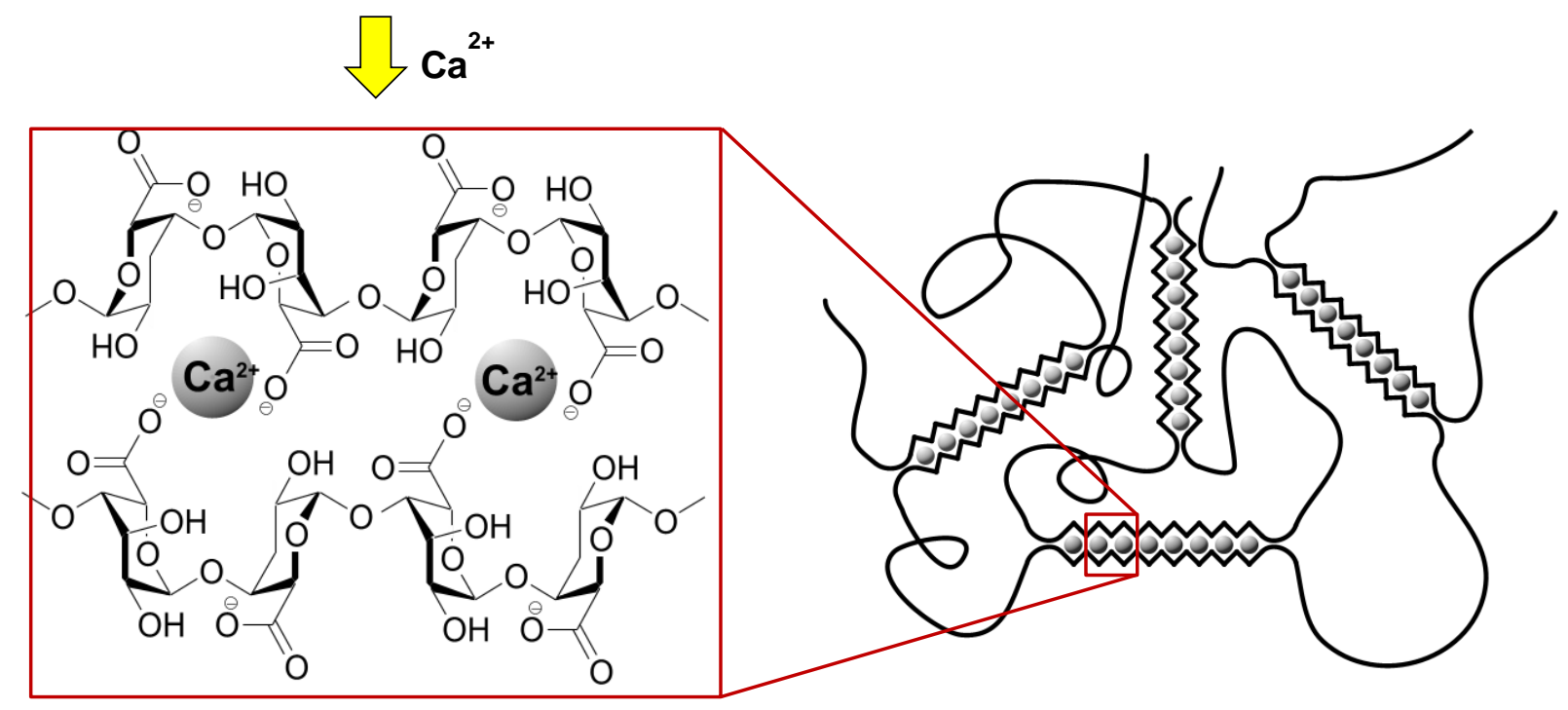

Figure 1. Structure of alginate and its binding of calcium cations in egg-box model.(Grant, Morris, Rees, Smith \& Thom, 1973)

We have previously reported on the controlled formation and dissolution of iron(III) cross-linked alginate hydrogels by manipulating the oxidation state of iron cations. Iron(II) cations in concentrations $25 \mathrm{mM}$ 
and below do not produce cross-linking of alginate chains and iron(II) salts can be dissolved in a solution of sodium alginate to form a homogeneous solution. Chemical or electrochemical oxidation of of iron(II) cations to iron(III) in the presence of sodium alginate results in the formation of the hydrogels allowing their deposition on a variety of conductive surfaces(Jin et al., 2011) while electrochemical reduction of iron(III) cations to iron(II) in the obtained hydrogel films results in their dissolution. The process of reduction can also be conducted photochemically using visible light and an $\alpha$-hydroxycarboxylic acid as a sacrificial electron donor. This photochemical reaction can be performed in spatially selective manner enabling photochemical patterning of iron(III) cross-linked alginate hydrogels.(Bruchet, Mendelson \& Melman, 2013; Narayanan, Melman, Letourneau, Mendelson \& Melman, 2012) The simplicity of this approach and very mild conditions of the photodegradation make iron(III) cross-linked alginate hydrogel a perspective biocompatible photoresist for preparation of sophisticated $2 \mathrm{D}$ and $3 \mathrm{D}$ patterned scaffolds for biomedical applications. (Machida-Sano, Matsuda \& Namiki, 2009; Machida-Sano, Ogawa, Ueda, Kimura, Satoh \& Namiki, 2012) However, in contrast to calcium cations, iron(III) cations form highly stable complexes with amino acids and phosphate ligands thus making iron(III) alginate hydrogels less stable in contact with growth media and body fluids containing these ligands. Furthermore, iron cations possess highly diverse physiological activity, and their presence may induce oxidative stress(Theil \& Goss, 2009; Valko, Rhodes, Moncol, Izakovic \& Mazur, 2006) in cells growing on iron(III) hydrogel support thus suggesting that calcium alginate hydrogels can be preferred for biomedical applications. In this paper we report on our study of reductive exchange of iron(III) cations in iron(III) cross-linked alginate hydrogels to $\mathrm{Ca}^{2+}$ which allows application of the abovementioned versatile methods of fabrication of patterned iron(III) cross-linked hydrogels for preparation of fully biocompatible calcium cross-linked alginate hydrogels.

\section{Materials and methods}

Materials and instruments. Alginic acid sodium salt from brown algae was purchased from Sigma (BioReagent, catalog number 71238 and Low Viscosity, catalog number A2158). Iron(II) chloride, 
iron(III) chloride, calcium chloride, calcium carbonate, 2,2'-bipyridine, gluconic acid, ascorbic acid, lactic acid and 2-hydroxyisobutyric acid were purchased from Fisher Scientific and used without further purification. Irradiation with visible light was done using $300 \mathrm{~mW} 405 \mathrm{~nm}$ laser. Light intensities were measured by Hioki 3664 optical power meter. Optical measurements were performed using Agilent 8453 UV-visible spectrophotometer. Circular dichroic spectra were recorded on a Jasco J-715 spectropolarimeter. Determination of concentration of iron cation by atomic absorption spectroscopy (AAS) were done using Perkin Elmer AAnalyst 200 Atomic Absorption Spectrometer using flame atomic absorption, using a Multi-Element Cu-Fe-Mn-Zn Lumina Hollow Cathode Lamp.

General procedure for preparation of iron(III) cross-linked alginate hydrogel. To a solution of sodium alginate in ultrapure water $(2.0 \% \mathrm{w} / \mathrm{v}, 3.0 \mathrm{~mL})$ was added dropwise $3.0 \mathrm{~mL}$ of a freshly prepared solution of iron(II) chloride in ultrapure water $(40 \mathrm{mM})$ under vigorous $(\geq 1000 \mathrm{rpm})$ stirring. The resultant homogeneous solution was transferred to a Petri dish $(30 \mathrm{~mm}$ in diameter, $1.5 \mathrm{~mL})$ or to one of transparent sides of quartz cuvette $(45 \mathrm{~mm} \times 12.5 \mathrm{~mm} \times 12.5 \mathrm{~mm}, 3.5 \mathrm{~mL})$ forming $1.0 \mathrm{~mm}$ layer that was oxidized by air for $48 \mathrm{~h}$ at $25{ }^{\circ} \mathrm{C}$ in a closed plastic container under $100 \%$ humidity. The progress of the oxidation was monitored by UV spectroscopy at $350 \mathrm{~nm}$. Substantial differences were found in optical absorption of hydrogels prepared from two different sources of sodium alginate. Typically, after $48 \mathrm{~h}$ of air oxidation the resultant samples of iron(III) alginate prepared under these conditions using the Low Viscosity alginate had an absorbance of $1.45 \pm 0.1$ at $350 \mathrm{~nm}$ and using Bioreagent alginate $1.0 \pm 0.2$ at $350 \mathrm{~nm}$. Unless otherwise stated, all subsequent experiments were conducted using Bioreagent grade sodium alginate.

Preparation of homogeneous calcium cross-linked hydrogel. Following modified Kuo's procedure(Kuo \& Ma, 2001), $1.5 \mathrm{~mL}$ of a solution of sodium alginate ( $2 \% \mathrm{w} / \mathrm{v})$ was vortexed with $0.75 \mathrm{~mL}$ of a suspension of $\mathrm{CaCO}_{3}$ in water $(80 \mathrm{mM})$ for one minute followed by vortexing with $0.75 \mathrm{~mL}$ of a solution of gluconolactone $(160 \mathrm{mM})$ for one minute. An aliquot $(0.9 \mathrm{~mL})$ of the resultant suspension was transferred to a Petri dish or a horizontally held quartz cuvette and left for hydrogel formation for $24 \mathrm{~h}$. 
Cation exchange in calcium cross-linked alginate hydrogel with iron(III) chloride. Six cuvettes containing $0.9 \mathrm{ml}$ of calcium crosslinked alginate hydrogel on their were prepared according to the standard procedure above. Three cuvettes were incubated with $1 \%$ aqueous iron(III) chloride for $30 \mathrm{~min}$; three other cuvettes were incubated with $0.1 \%$ aqueous iron(III) chloride for $200 \mathrm{~min}$ (solution changed after 20, 70 and $150 \mathrm{~min}$ ). Before measurements the iron(III) solutions were removed from the cuvettes, replaced with distilled water for absorbance measurement, and returned to cuvettes. After completion of the cation transfer, the hydrgels were removed from the cuvettes, dried with a paper towel and placed in centrifuge tubes. The gels were dissolved by adding $3 \mathrm{ml}$ of disodium EDTA $(125 \mathrm{mM})$ in the centrifuge tubes and warming up to $50^{\circ} \mathrm{C}$ for $1 \mathrm{~h}$. The tubes were left to cool to room temperature. $7 \mathrm{ml}$ of a $5 \%$ solution of $\mathrm{HNO}_{3}$ was added to the tubes before centrifuging for $10 \mathrm{~min}$ at $1600 \mathrm{xG}$ to separate the alginate from the solutions. The supernatant was removed and the precipitate washed with $9 \mathrm{ml}$ of $5 \%$ $\mathrm{HNO}_{3}$ and centrifuged for $10 \mathrm{~min}$. For each sample, the solutions extracted from the two centrifugations were combined and the volume brought up to $50 \mathrm{ml}$ using $5 \% \mathrm{HNO}_{3}$ solution. The concentration of iron in each sample was determined by flame AAS to calculate the extinction coefficient.

General procedure for exchange of iron(III) cations to $\mathrm{Ca}^{2+}$ by ascorbate reduction. An iron(III) crosslinked hydrogel (1 mm thick) was created in a Petri dish or in a horizontally positioned quartz cuvette. In the case of Petri dish after $48 \mathrm{~h}$ of air oxidation was topped with $5.0 \mathrm{~mL}$ of a solution of sodium ascorbate or ascorbic acid (25-50 $\mathrm{mM})$ and calcium chloride (25-50 mM). The dish was gently shaken using an orbital shaker (120 min, $50 \mathrm{rpm}$ ). For each UV-Visible measurement of progress of cation exchange the solution phase was decanted and the Petri dish was kept on side while the absorbance of the hydrogel was measured. After the measurement, this solution phase was added back to the Petri dish. For hydrogels prepared in a quartz cuvette, after $48 \mathrm{~h}$ of air oxidation the quartz cuvette was positioned vertically and filled with the solution of ascorbic acid $(50 \mathrm{mM})$ and calcium chloride $(50 \mathrm{mM})$, up to the top of the cuvette. For each UV-Visible measurement, the solution was removed and the cuvette filled with 
ultrapure water. After measurement, the water was removed and the solution was placed back in the cuvette to continue until $3 \mathrm{~h}$ of irradiation accumulated.

General procedure for photochemical reductive exchange of iron(III) cations to $\mathrm{Ca}^{2+}$ in a solution of lactic or 2-hydroxybutyric acid. A hydrogel prepared according to the general procedure (1 $\mathrm{mm}$ thickness) after $48 \mathrm{~h}$ of air oxidation was topped with $5.0 \mathrm{~mL}$ of solution of lactic acid $(25 \mathrm{or} 50 \mathrm{mM})$ and calcium chloride (25 or $50 \mathrm{mM}$ ). The hydrogel was irradiated by diffused $405 \mathrm{~nm}$ laser beam (44 $\mathrm{mW} / \mathrm{m}^{2}$ ). The irradiation was periodically stopped to record UV-Vis spectra. For each UV-Vis measurement, the solution was removed and the cuvette filled with ultrapure water. After measurement, water was replaced with the solution containing lactic acid and calcium chloride.

General procedure for photochemical reductive exchange of iron(III) cations to $\mathrm{Ca}^{2+}$ using hydrogel containing sodium lactate. To a solution of sodium alginate in ultrapure water $(2.0 \% \mathrm{w} / \mathrm{v}, 1.0 \mathrm{~mL}) \mathrm{was}$ added dropwise $0.92 \mathrm{~mL}$ of a freshly prepared solution of iron(II) chloride in ultrapure water (43.48 mM), and $0.08 \mathrm{~mL}$ of a $500 \mathrm{mM}$ solution of sodium lactate $(\mathrm{pH}=7)$, under vigorous stirring $(\geq 1000 \mathrm{rpm})$. From the resultant homogeneous solution, $0.9 \mathrm{~mL}$ was transferred to a quartz cuvette $(45 \mathrm{~mm} \times 12.5 \mathrm{~mm} \times$ $12.5 \mathrm{~mm}, 3.5 \mathrm{~mL}$ ) in a way to cover one of the transparent sides of the cuvette with a $1.0 \mathrm{~mm}$ layer of the solution to oxidize for $48 \mathrm{~h}$ at $25{ }^{\circ} \mathrm{C}$ and $100 \%$ humidity. After $48 \mathrm{~h}$ of air oxidation, the quartz cuvette was put vertically and filled with the solution of calcium chloride $(50 \mathrm{mM})$, up to the top of the cuvette and the side covered by the hydrogel was irradiated with $405 \mathrm{~nm}$ laser focused to give a light intensity of $44 \mathrm{~mW} / \mathrm{m}^{2}$ in the center for $12 \mathrm{~h}$.

Determination of concentration of calcium cations in the hydrogel after reductive cation exchange. After reductive exchange, calcium alginate in a quartz cuvette was dissolved using $1 \mathrm{~mL}$ of a $0.125 \mathrm{mM}$ ethylenediaminetetraacetate (EDTA) standardized solution $(\mathrm{pH}=10)$. After the dissolution the volume of the solution was adjusted to $10 \mathrm{~mL}$ using distilled water and the $\mathrm{pH}$ was adjusted to $10 \mathrm{using} 1 \mathrm{~mL}$ of concentrated ammonia buffer ( $90 \mathrm{~g} \mathrm{NH}_{4} \mathrm{Cl}$ and $375 \mathrm{~mL} \mathrm{NH}_{4} \mathrm{OH}(28-30 \%)$ diluted to $500 \mathrm{~mL}$ in water). 
The excess of EDTA was back titrated using a standardized solution of calcium chloride, the equivalence determined in the presence of Eriochrome Black T ( 2 drops of a $1 \%$ alcoholic solution).(Ray Sarkar \& Chauhan, 1967)

Monitoring the progress of photochemical cation exchange by CD spectroscopy. An iron(III) cross-linked alginate hydrogel previously prepared in a quartz cuvette according to the general procedure after $48 \mathrm{~h}$ of air oxidation was placed vertically and filled with water up to the top. The CD spectrum of the hydrogel was measured for the range 190-250 nm. After measurement water was removed and the cuvette filled with a solution of lactic acid $(50 \mathrm{mM})$ and calcium chloride $(50 \mathrm{mM})$, up to the top of the cuvette. The side opposite to the one covered by the hydrogel was irradiated with $405 \mathrm{~nm}$ laser $\left(44 \mathrm{~mW} / \mathrm{m}^{2}\right)$. After $0.25,0.5,1,2,3$, and $4 \mathrm{~h}$ of irradiation, the solution was removed by decanting and the cuvette filled with ultrapure water to record $\mathrm{CD}$ spectra $(100 \mathrm{~nm} / \mathrm{min})$. After each measurement water was removed and replaced with the solution of lactic acid and calcium chloride, and the irradiation resumed.

Measurement of CD spectra of calcium cross-linked hydrogels obtained by dissolution of calcium carbonate and by cation exchange of iron(III). A sample of calcium alginate sample prepared as described above in a cuvette by reductive exchange with lactic acid present in solution and a calcium alginate sample prepared in a cuvette by reductive exchange with sodium lactate present in the hydrogel were treated with a solution of 2,2'-bipyridine $(25 \mathrm{mM})$ and $\mathrm{CaCl}_{2}(50 \mathrm{mM})$ for $2 \mathrm{~h}$, to remove any remaining iron(II) cations. The supernatant was removed, the hydrogels washed with ultrapure water 5 times and then incubated with a $0.5 \%$ solution of calcium chloride until the hydrogel showed no peak of Fe(bipy) $)_{3}$ complex at $520 \mathrm{~nm}$.

Fabrication for photochemically patterned calcium cross-linked alginate hydrogel. An iron(III)-alginate hydrogel, containing $25 \mathrm{mM}$ iron(II) chloride, $20 \mathrm{mM}$ sodium lactate and $1 \% \mathrm{w} / \mathrm{v}$ sodium alginate, was prepared in a Petri dish and left for air oxidation for $48 \mathrm{~h}$. The hydrogel was irradiated through a photographic mask with $405 \mathrm{~nm}$ laser at $44 \mathrm{~mW} / \mathrm{m}^{2}$ for $6 \mathrm{~min}$, following the same procedure previously 
described for the photodegradation of iron(III) alginate hydrogels.(Narayanan, Melman, Letourneau, Mendelson \& Melman, 2012) The hydrogel was then washed with a $\mathrm{NaCl}$ solution (0.9\%) on an orbital shaker $(50 \mathrm{rpm})$ for $10 \mathrm{~min}$. The resulting patterned iron(III) crosslinked alginate hydrogel was kept under $100 \%$ humidity. A solution of calcium chloride $(50 \mathrm{mM})$ and ascorbic acid $(50 \mathrm{mM})$ was poured in the Petri dish, covering the patterned hydrogel, and left for shaking on an orbital shaker (50 rpm) for $10 \mathrm{~min}$. The solution was removed and the hydrogel slightly washed with ultrapure water.

Preparation of dansyl-labeled sodium alginate. To a mixture of N-dansyl 1,3-diaminopropane(Bhoi et al., 2014) (1.75 mL of $57 \mathrm{mM}$ solution in water) $0.1 \mathrm{mmol})$, sodium alginate $(0.2 \mathrm{~g}, 10 \mathrm{~mL}$ of $2 \% \mathrm{w} / \mathrm{v}$ solution in water ), $\mathrm{N}$-hydroxybenzotriazole (HOBT, $1.75 \mathrm{~mL}$ of $57 \mathrm{mM}$ solution in water, $0.1 \mathrm{mmol}$ ) at 0 ${ }^{\circ} \mathrm{C}$ under stirring was added $\mathrm{EDC}(6.5 \mathrm{~mL}$ of $62 \mathrm{mM}$ solution in water, $0.4 \mathrm{mmol})$ and the resultant solution was stirred for $48 \mathrm{~h}$ at room temperature. The polymer was precipitated with $95 \%$ ethanol, filtered, and dried. The residue was redissolved in water and the precipitation was repeated twice. The resultant residue was redissolved in water and lyophilized.

Procedure for deposition of a film of calcium cross-linked alginate hydrogel on graphite electrode. To a mixture of dansyl-labeled sodium alginate $(625 \mathrm{uL}$ of $2 \%$ solution in water), sodium alginate $(1.875 \mathrm{~mL}$ of $2 \%$ solution in water), sodium sulfate $(1 \mathrm{~mL}$ of $0.5 \mathrm{M}$ solution in water), was added dropwise under vigorous stirring a solution of iron(II) sulfate $(1.5 \mathrm{~mL}$ of $66.7 \mathrm{mM}$ solution in water). The resultant homogeneous mixture was sonicated to remove air bubbles and used as electrolyte for deposition of iron(III) alginate hydrogel on $0.7 \mathrm{~mm}$ graphite anode. The deposition was conducted under constant potential $+0.8 \mathrm{~V}$ for 20 min followed by removal of the anode coated with the hydrogel and rinsing it with water. The coated anode was incubated in a solution containing $50 \mathrm{mM}$ of calcium chloride and $50 \mathrm{mM}$ of sodium lactate for $90 \mathrm{~min}$ followed by $2 \mathrm{~h}$ irradiation at $44 \mathrm{~mW} / \mathrm{cm}^{2}$ of $405 \mathrm{~nm}$ blue laser.

\section{Results and discussions}


The strategy of using advanced methods for fabrication of calcium alginate hydrogel films or coatings from iron(III) alginate required finding an efficient way for replacing iron(III) to $\mathrm{Ca}^{2+}$ cations that does not damage or distort hydrogel structure and patterning obtained through electrochemical deposition or photochemical patterning. Below we studied several approaches to cations exchange in a homogeneous iron(III) cross-linked hydrogel including a direct exchange, an exchange in the presence of a chemical reductant, and a photochemical exchange in the presence of a sacrificial reductant. The obtained results were further applied to conducting the cation exchange in electrochemically deposited and photochemically patterned iron(III) alginate films.

3.1 Direct exchange of calcium and iron(III) cations in alginate hydrogels. Our initial attempts for inducing cation exchange in iron(III) cross-linked alginate hydrogel involved its incubation with a large excess of calcium chloride. The hydrogel was prepared by air oxidation of a solution containing sodium alginate $(1 \mathrm{w} / \mathrm{v} \%)$ and iron(II) chloride $(25 \mathrm{mM})$ in a Petri dish. The air oxidation produced $1 \mathrm{~mm}$ thick layer of a homogeneous transparent yellow hydrogel within 48 h.(Narayanan, Melman, Letourneau, Mendelson \& Melman, 2012) Iron(III) cations in the hydrogel provided a strong light absorbance below $450 \mathrm{~nm}$ allowing easy monitoring of their concentration in the hydrogel. The hydrogel was treated with $50 \mathrm{mM}$ solution of calcium chloride. After $48 \mathrm{~h}$ of the treatment no changes in UV-Vis spectra of the hydrogel were observed thus indicating that no appreciable cation transfer occurred. The absence of the cation exchange can be attributed to a higher thermodynamic stability iron(III) cross-linked alginate hydrogel vs. calcium alginate one, as has been reported previously by Whitesides at al.(Bracher, Gupta \& Whitesides, 2009) Alternatively, the absence of cation exchange could also be explained by an unusual kinetic stability of iron(III) alginate hydrogel. To determine the kinetics of cation exchange, we explored the opposite reaction of replacement of calcium cations in homogeneous calcium cross-linked alginate hydrogel to iron(III) cations. The calcium cross-linked hydrogel was prepared by a slow reaction of 20 $\mathrm{mM}$ calcium carbonate with an aqueous solution containing $1 \% \mathrm{w} / \mathrm{v}$ of sodium alginate and $40 \mathrm{mM}$ of gluconolactone.(Kuo \& Ma, 2001) The obtained hydrogel was treated with a $0.1 \%$ w/v or $1 \%$ w/v 
solution of iron(III) chloride was monitored by UV absorption of the alginate hydrogel at $400 \mathrm{~nm}$ after washing the hydrogel with water prior to every measurement. As can be seen from Fig 2, under these conditions the exchange of calcium cations to iron(III) proceeded rapidly with iron(III) cation absorption at $400 \mathrm{~nm}$ reaching 1.8 after $25 \mathrm{~min}$ of incubation with $1 \% \mathrm{w} / \mathrm{v}(37 \mathrm{mM})$ or after $65 \mathrm{~min}$ for $0.1 \% \mathrm{w} / \mathrm{v}$ (3.7 mM) iron(III) chloride solution.

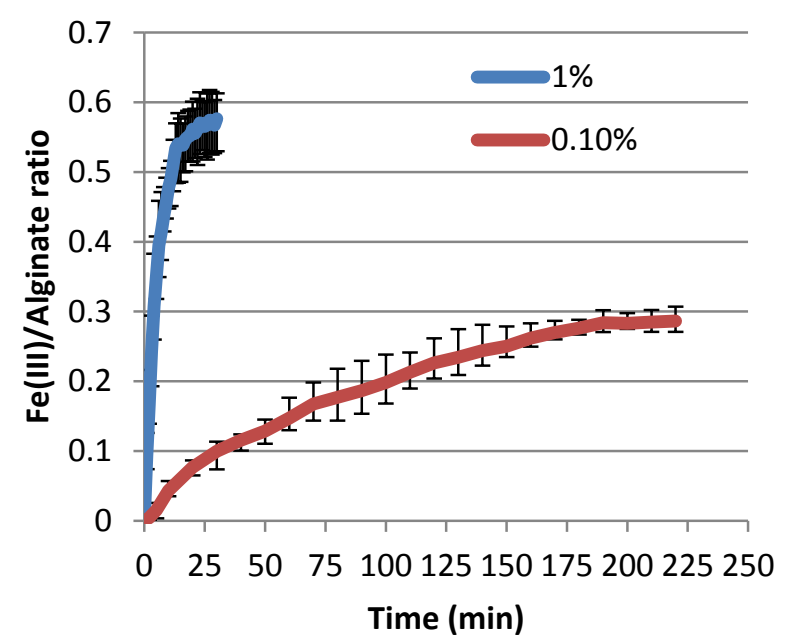

Figure 2. Kinetics of exchange of $\mathrm{Ca}^{2+}$ to iron(III) cations in calcium cross-linked alginate hydrogel by treatment with $1 \%$ or $0.1 \%$ of aqueous solutions iron(III) chloride.

3.2. Reductive exchange of iron(III) cations. The obtained results with direct cation exchange are not surprising as iron(III) is a typical "hard" metal cation that strongly binds "hard" ligands such as carboxylate groups of guluronate and/or mannuronate subunits of alginate. However, as we have shown recently,(Narayanan, Melman, Letourneau, Mendelson \& Melman, 2012) a reduction of iron(III) to iron(II) can result in a substantial weakening of iron cation binding to carboxylate groups of alginate chains suggesting that exchange of iron(II) cations to $\mathrm{Ca}^{2+}$ can be possible in the presence of a reducing agent. To explore the possibility of the reductive cation exchange we used homogeneous iron(III) alginate hydrogel obtained from a $48 \mathrm{~h}$ air oxidation of a solution containing iron(II) chloride (25 mM) and 
sodium alginate $(1 \% \mathrm{w} / \mathrm{v})$. A $1.0 \mathrm{~mm}$ thick layer of the hydrogel in Petri dish was exposed to a solution containing $25-50 \mathrm{mM}$ of ascorbic acid and $25-50 \mathrm{mM}$ of calcium chloride.

The treatment resulted in rapid changes in UV-Vis spectra of the hydrogel. Dependence of the changes in absorption at $350 \mathrm{~nm}$ from time is shown on Fig. 3, Left for all four these experiments. After $10 \mathrm{~min}$ the hydrogel become essentially colorless indicating reduction of iron(III) cations while retaining its size and overall shape, presumably because $\mathrm{Ca}^{2+}$ cations cross-links the alginate binding sites vacated by the resultant iron(II) cations. The rate of the reaction was substantially increased with doubling concentration of ascorbic acid from $25 \mathrm{mM}$ to $50 \mathrm{mM}$ but showed relatively minor variations to doubling of concentration of $\mathrm{Ca}^{2+}$ thus suggesting that the reduction of iron(III) cations by ascorbate is the rate limiting step of the process. The reductive exchange proceeded under weakly acidic to neutral conditions (pH 4 - 7.0). However, at $\mathrm{pH} 6$ and above the reaction is slowed down (Fig. 3, right) and is accompanied by formation of a polynuclear purple complexes of iron(II) cations with ascorbate.(Kim, Feng \& Lippard, 2007).
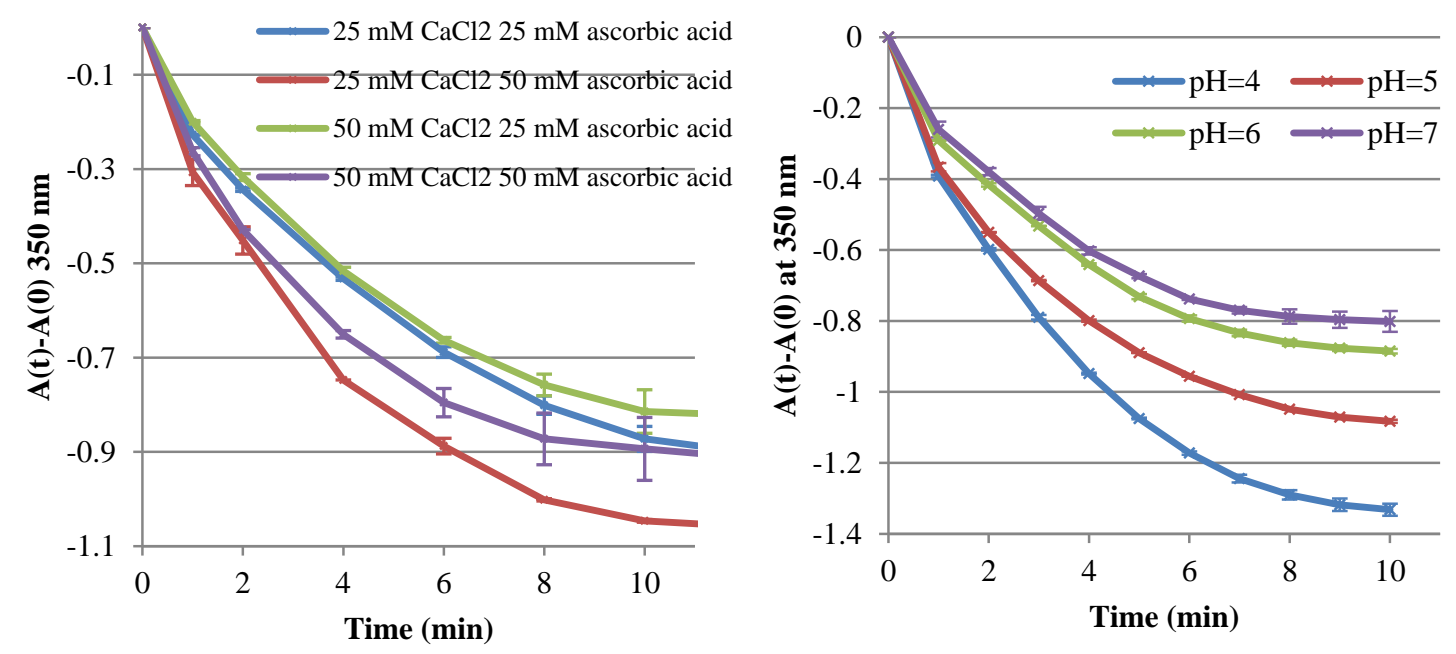
Figure 3. Left: Kinetics of reductive cation exchange in iron(III) cross-linked alginate hydrogel (low viscosity alginate) caused by treatment with 25-50 $\mathrm{mM}$ of ascorbic acid and 25-50 $\mathrm{mM}$ of calcium chloride measured as decrease of absorption at $350 \mathrm{~nm}$. Right: Kinetics of the reductive cation exchange with calcium chloride (25 $\mathrm{mM})$ and ascorbic acid (50 $\mathrm{mM})$ at $\mathrm{pH} 4$ to 7.

These results demonstrate that ascorbate induced reduction of iron(III) cations to iron(II) is accompanied by formation of colorless calcium cross-linked alginate hydrogel through exchange of iron(II) cations to $\mathrm{Ca}^{2+}$ as iron(II) cations at these concentrations do not bind carboxylate groups of alginate chains to induce cross-linking and hydrogel formation. However, calcium cross-linked hydrogel obtained in reduction of under all conditions was not completely transparent and showed minor light scattering which indicated its microheterogeneity. The microheterogeneity can be caused by several factors such as faster diffusion of ascorbate in comparison to calcium cations which can result in partial dissolution of alginate hydrogel followed by its reconstitution with calcium cations.

3.3 Photoinduced reductive exchange of iron(III) cations to $\mathrm{Ca}^{2+}$. To overcome the problem of microheterogeneity of calcium alginate hydrogel obtained with ascorbic acid/calcium chloride cation exchange we explored alternative methods of reduction of iron(III) cations using a photoinduced reductive cation exchange in iron(III) cross-linked alginate hydrogel with lactic acid as a sacrificial photoreductant. A homogeneous iron(III) cross-linked hydrogel was prepared by air oxidation (48 hours) of a solution containing $20 \mathrm{mM}$ of iron(II) chloride and $1 \% \mathrm{w} / \mathrm{v}$ of sodium alginate. The obtained homogeneous hydrogel was immersed into a solution containing $25-50 \mathrm{mM}$ of calcium chloride and 25$50 \mathrm{mM}$ of lactic acid and irradiated through the layer of the solution with a $405 \mathrm{~nm}$ diffused laser beam with intensity $44 \mathrm{~mW} / \mathrm{cm}^{2}$. The kinetics of iron(III) cation reduction was monitored spectrophotometrically at $350 \mathrm{~nm}$ (Fig. 4) after temporary taking out the hydrogel gel from the solution of lactic acid. As expected, the irradiation produced continuous decrease in absorbance of iron(III) cations at 
$350 \mathrm{~nm}$ and produced colorless hydrogels after $11 \mathrm{~min}$ of irradiation thus indicating complete disappearance of iron(III) cations. The resultant transparent homogeneous hydrogel was washed with 25 $\mathrm{mM}$ solution of 2,2'-bipyridine to remove any traces of remaining iron(II) and then with distilled water to until complete disappearance of purple color of iron(II)- 2,2'-bipyridine complex. To quantify the amount of calcium cations after the cation exchange a sample of the resultant hydrogel was dissolved in EDTA followed by titrimetric determination which indicated $21.6 \mathrm{mM}$ concentration of $\mathrm{Ca}^{2+}$. Taking into account that concentration of carboxylate groups in the $1 \%$ hydrogel $51.5 \mathrm{mM}$ the $\mathrm{Ca}^{2+}$ :carboxylate ratio is 0.41 , not far away from the theoretical ratio of $1: 2$.

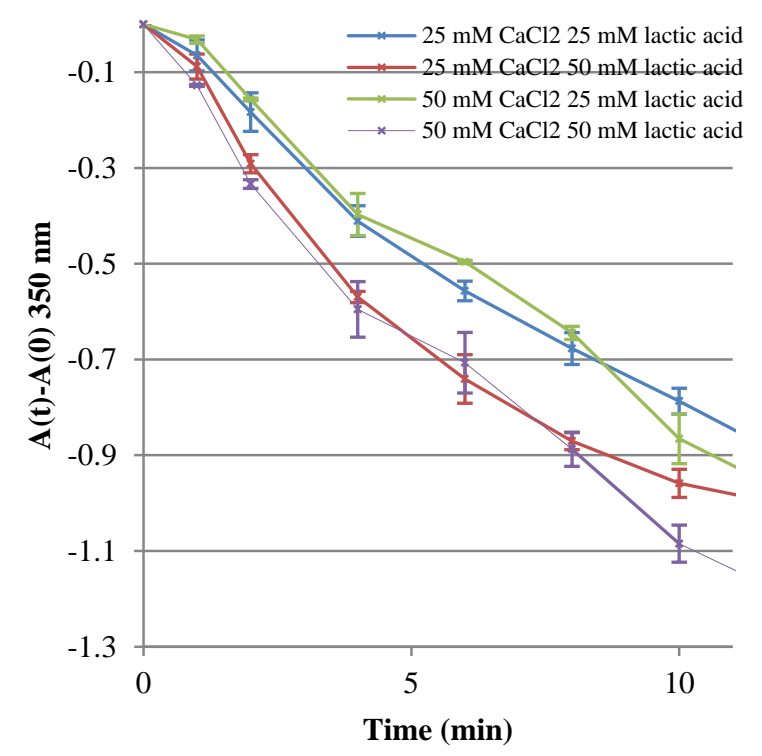

Figure 4. Kinetics of photoreductive ion exchange in iron(III) cross-linked alginate hydrogels containing 25-50 mM lactic acid in a solution of 25-50 mM of calcium chloride

3.4 Photoinduced reductive exchange of iron(III) cations to $\mathrm{Ca}^{2+}$ in electrochemically deposited iron(III) cross-linked hydrogel. The approach of photoinduced reductive cation transfer was then applied to a thin film of iron(III) cross-linked alginate hydrogel obtained by electrochemical deposition on graphite surface. The electrochemical oxidation (Jin et al., 2011) forms films of iron(III) alginate which can serve as a universal approach toward deposition of a hydrophilic biocompatible coating on essentially any 
conductive surface. To study formation of thin films of transparent alginate hydrogel we prepared sodium alginate carrying fluorescent dansyl functionality. The dansyl group was introduced through EDC coupling of sodium alginate with $N$-dansyl 1,3-diaminopropane. The obtained fluorescent alginate was used for visualization of its deposition on a graphite rod using our published procedure.(Jin et al., 2011) The process of the electrochemical alginate deposition at a constant potential $+0.8 \mathrm{~V}$ for 20 min in electrolyte containing a solution of fluorescent alginate, iron(II) sulfate, and sodium sulfate. The yellow layer of iron(III) alginate hydrogel on the electrode lacked any visible fluorescence due to its quenching by iron(III) cations. The coated electrode was transferred into a solution of calcium chloride and sodium lactate followed by irradiation with $405 \mathrm{~nm}$ laser for $2 \mathrm{~h}$. As expected, the reductive cation exchange and removal of iron cations from the hydrogel resulted in a colorless hydrogel possessing a visible fluorescence (Fig. 5) demonstrating the successful exchange of iron(III) cations to $\mathrm{Ca}^{2+}$. These results suggest that the electrochemical deposition can be used for coating of essentially any conductive surface with a thin layer of calcium alginate hydrogel

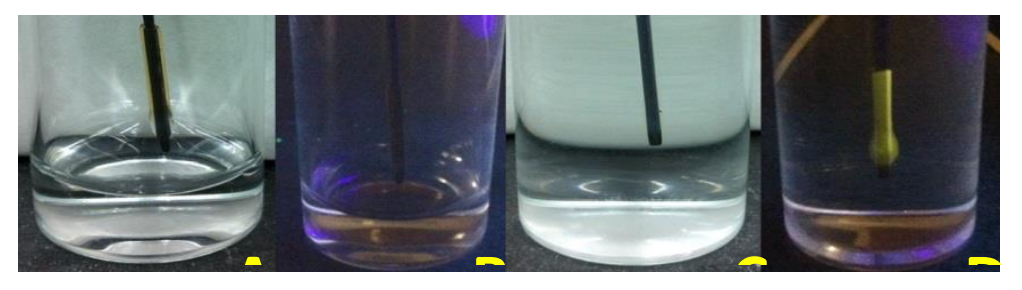

Figure 5. Preparation of calcium cross-linked hydrogel coating through photoinduced reductive cation exchange of electrochemically deposited iron(III) cross-linked alginate hydrogel. (A) iron(III) crosslinked hydrogel deposited on graphite electrode at visible light; (B) the same under $254 \mathrm{~nm} U$ V light; (C) film of calcium alginate on the electrode after photochemical reduction, visible light; $(D)$ the same under UV light.

3.5 Study of the structure of $\mathrm{Ca}^{2+}$ cross-linked alginate hydrogel by CD spectroscopy. Successful preparation of homogeneous $\mathrm{Ca}^{2+}$ cross-linked alginate hydrogels through reductive cation exchange in iron(III) cross-linked hydrogel raised the question of the mode of binding of calcium cations in the 
obtained hydrogels. Binding of $\mathrm{Ca}^{2+}$ cations in hydrogels obtained by a reaction of sodium alginate occurs predominantly between polyguluronate domains of different alginate chains to form egg-box structures (Fig. 1).(Grant, Morris, Rees, Smith \& Thom, 1973; Sikorski, Mo, Skjak-Braek \& Stokke, 2007) The egg-box arrangement has a specific signature in circular dichroism (CD) spectra with a positive band at around $205 \mathrm{~nm}$ and a smaller negative band at around $220 \mathrm{~nm}$ (Morris, Rees, Thom \& Boyd, 1978).

In contrast, little is known about the arrangement of iron(III) cations with guluronate and mannuronate residues. Because of the difference in coordination numbers of iron(III) and $\mathrm{Ca}^{2+}$ cations and their radii a considerable difference in binding mode can be observed. The process of the reductive cation exchange of iron(III) to $\mathrm{Ca}^{2+}$ cations can either retain the original binding mode or produce the thermodynamically stable egg-box arrangement. To determine the mode of binding in calcium cross-linked hydrogels obtained by reductive cation exchange we measured $\mathrm{CD}$ spectra of these hydrogels and monitored its transformation during the cation exchange.

The reductive cation exchange was performed using the photochemical reaction with lactic acid as a sacrificial reductant. The reaction was conducted with a sample of iron(III) cross-linked alginate hydrogel containing $20 \mathrm{mM}$ of sodium lactate in the hydrogel, and with another sample containing the hydrogel only. A $1.0 \mathrm{~mm}$ layer of the hydrogel was prepared through $48 \mathrm{~h}$ oxidation of an aqueous solution containing iron(II) chloride in $1 \% \mathrm{w} / \mathrm{v}$ of sodium alginate on a transparent wall of a quartz cuvette. The cuvette was filled with a solution containing $50 \mathrm{mM}$ of calcium chloride, $50 \mathrm{mM}$ of lactic acid, and irradiated at $44 \mathrm{~mW} / \mathrm{cm}^{2}$ for $3 \mathrm{~h}$, resulting in exchange of iron(III) cations to $\mathrm{Ca}^{2+}$. Samples were treated with an $50 \mathrm{mM}$ solution 2,2'-bypiridine and $50 \mathrm{mM} \mathrm{CaCl}_{2}$ to remove any remaining iron(II) cations followed by washing with $0.5 \% \mathrm{w} / \mathrm{v}$ solution of $\mathrm{CaCl}_{2}$ until no absorption of iron(II)- 2,2'-bypiridine complex at $525 \mathrm{~nm}$ is observed. The control experiment involved preparation of a layer of calcium crosslinked alginate hydrogel through slow dissolution of a suspension containing calcium carbonate (20 mM) and $40 \mathrm{mM}$ of gluconolactone in $1 \% \mathrm{w} / \mathrm{v}$ solution of sodium alginate.(Kuo \& Ma, 2001) 
The CD spectra of these hydrogels obtained after complete exchange of iron with calcium cations as well of the control sample are shown on Fig. 6. As can be seen from these spectra, photochemical reductive cation exchange in both samples of these hydrogels resulted in formation of a strong positive CD peak at $205 \mathrm{~nm}$ and smaller negative peak at $220 \mathrm{~nm}$. Recorded CD spectrum of the control experiment that involved formation of calcium alginate hydrogel by dissolution of calcium carbonate had very similar peaks thus strongly suggesting that all three calcium alginate hydrogel samples possess similar egg-box binding arrangements.

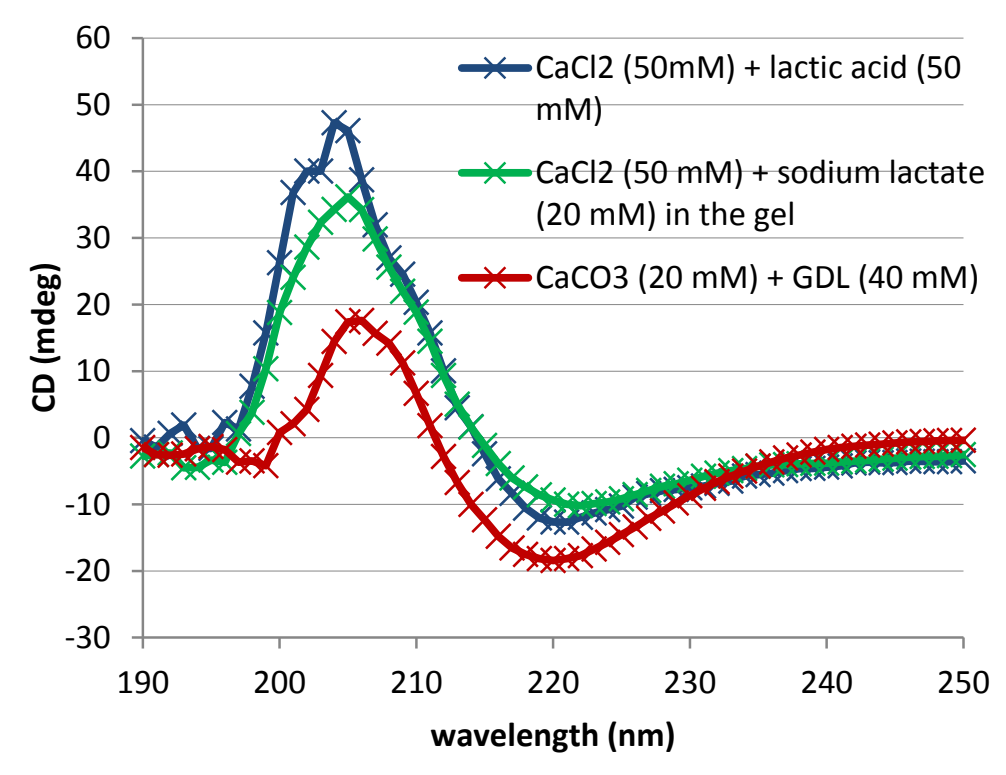

Figure 6. Comparison of CD spectra of hydrogels obtained by irradiation of samples of iron(III) crosslinked alginate hydrogels immersed into solutions containing $50 \mathrm{mM}$ of calcium chloride and $50 \mathrm{mM}$ of lactic acid (green and blue) with that of calcium cross-linked alginate hydrogel (red) prepared according to the published procedure.

To study the kinetics of the reductive cation exchange and formation of calcium alginate hydrogel these experiments were repeated using a sample of the same iron(III) cross-linked alginate hydrogel with periodical interruption of the $405 \mathrm{~nm}$ irradiation to measure CD spectra of the hydrogel. After 15, 30, 60, 120,180 , and $240 \mathrm{~min}$ of irradiation the $1 \mathrm{~mm}$ thick sample of the hydrogel was transferred into $\mathrm{CD}$ 
spectrometer and spectra from 190 to $250 \mathrm{~nm}$ were recorded (Fig. 7). Monitoring the CD spectra was complicated by a strong light absorption of remaining iron(III) cations in the hydrogel which produced very noisy $C D$ signal, especially at the beginning of irradiation when their concentration was maximal. As a result, little information can be obtained on the binding mode of the initial iron(III) cross-linked hydrogel. However, the progress of the photochemical cation exchange clearly showed growth of a strong positive CD peak near $210 \mathrm{~nm}$ and a weak negative peak near $235 \mathrm{~nm}$ that very much resembled analogous peaks in Fig. 6. Based on these results it can assumed that the process of exchange iron(III) cation results in immediate formation of the egg-box binding mode in the resultant $\mathrm{Ca}^{2+}$ alginate hydrogel.

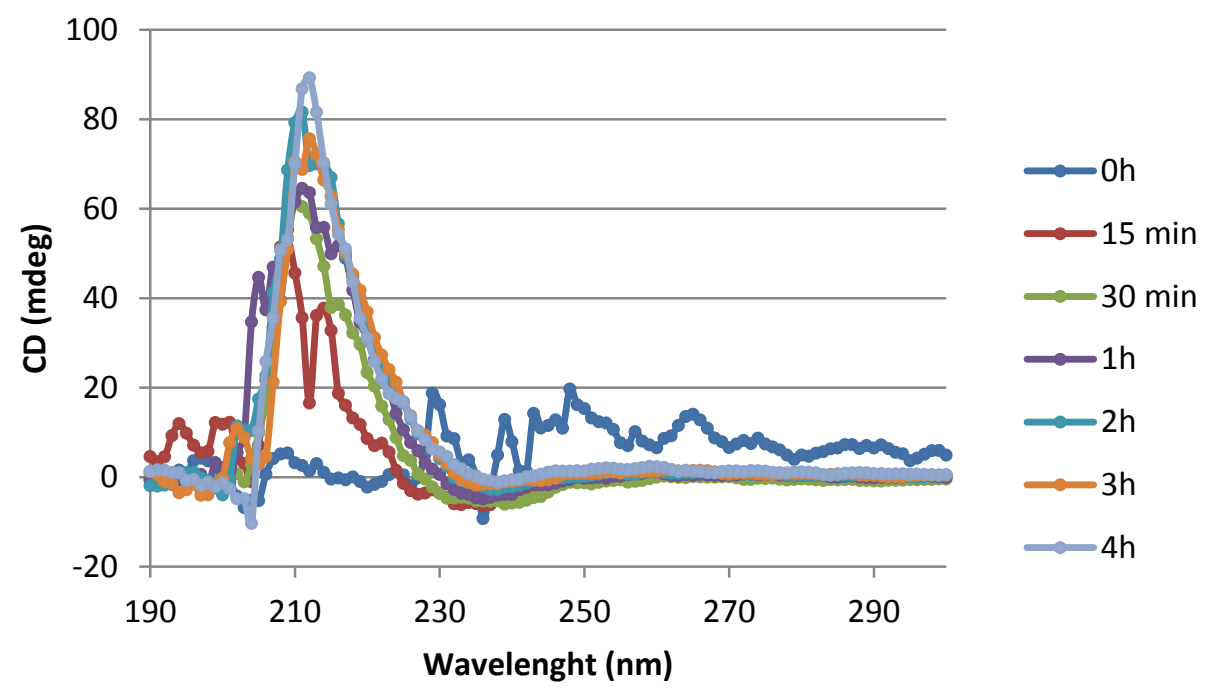

Figure 7. CD spectra of calcium alginate hydrogel obtained by (a) photochemical reduction with $50 \mathrm{mM}$ of lactic acid and $50 \mathrm{mM} \mathrm{CaCl}$; (b) photochemical reduction with $50 \mathrm{mM} \mathrm{CaCl}$ on a hydrogel containing $20 \mathrm{mM}$ sodium lactate and (c) treatment of sodium alginate with calcium carbonate/gluconolactone.

3.6 Biocompatibility of the obtained calcium alginate hydrogels and their stability in growth media. The observed similarity of calcium cross-linked alginate hydrogels obtained through 
cation exchange of iron(III) cross-linked hydrogel and through direct reaction of alginate with calcium cations indicates the identity of their biological properties. The extensive use of calcium cross-linked alginate hydrogels obtained by the latter method for cells encapsulation and tissue engineering has revealed their biocompatibility. It has been reported that hydrogels prepared from different alginate sources showed the lack of cytotoxicity and very small mitogenic activity.(Orive, Carcaboso, Hernandez, Gascon \& Pedraz, 2005) Purified alginates of biochemical grades are very weak inducers of inflammatory response.(Orive, Carcaboso, Hernandez, Gascon \& Pedraz, 2005) High biocompatibility of calcium alginate hydrogels in vitro and in vivo has been demonstrated in numerous experiments involving regeneration of cartilage(Kisiday et al., 2002; Re'em, Tsur-Gang \& Cohen, 2010; Wang, Yang, Lin, Liu, Liu \& Lin, 2012), bones(Alsberg, Anderson, Albeiruti, Franceschi \& Mooney, 2001; Grellier et al., 2009; Tang, Chen, Weir, Thein-Han \& Xu, 2012), and other organs(Ruvinov, Leor \& Cohen, 2011; Yu et al., 2010) using alginate scaffolds. Supplementing these data we investigated the stability of obtained alginate hydrogels in commonly used growth media. Because of the presence of univalent metal cations and chelators in growth media ionically cross-linked alginate hydrogel can undergo slow dissolution. To determine stability of iron(III) and calcium crosslinked hydrogel we prepared four samples of homogeneous hydrogels: (A) with homogeneous iron(III) cross-linked hydrogel prepared by air oxidation of iron(II) alginate; (B) iron(III) crosslinked hydrogel prepared by exchange of calcium alginate with iron(III) cations; (C) calcium alginate obtained from hydrogel A through photochemical cation exchange with calcium chloride and lactate; and (D) calcium cross-linked alginate hydrogel obtained by reaction of sodium alginate. After incubation of these samples with a large excess of RPMI and MEM growth media at $25^{\circ} \mathrm{C}$ for 20 hours iron(III) cross-linked hydrogel A was completely dissolved 
in both RPMI and MEM media. Iron(III) cross-linked hydrogel B was stable in MEM but was dissolved in RPMI in 48 h. In contrast, both calcium cross-linked alginate hydrogels C and D were fully stable in both RPMI and MEM media for at least four days. The difference in stability of iron(III) and calcium hydrogels can be explained by substantially stronger binding of iron(III) vs. calcium cations by chelate ligands such as amino acids, phosphate, and polyphosphate that are present in growth media.

3.7Fabrication of patterned $\mathrm{Ca}^{2+}$ cross-linked alginate hydrogel. These experiments demonstrate facile exchange of iron(III) cations in alginate hydrogels to $\mathrm{Ca}^{2+}$ using photochemical reduction with lactic acid. Integrating previously described methods of photochemical patterning of iron(III) cross-linked alginate hydrogels and the approach of cation exchange we developed a new simple and reproducible way for fabrication of photochemically patterned $\mathrm{Ca}^{2+}$ cross-linked hydrogels. To prove the feasibility of this approach (Fig. 8, above) we used $1 \mathrm{~mm}$ thick layer of iron(III) cross-linked alginate hydrogel containing $20 \mathrm{mM}$ of sodium alginate. After $48 \mathrm{~h}$ of air oxidation the hydrogel was photochemically patterned using diffused beam of $405 \mathrm{~nm}$ laser passing through a transparent PETF sheet with black letters deposited on it using a regular laser printer.
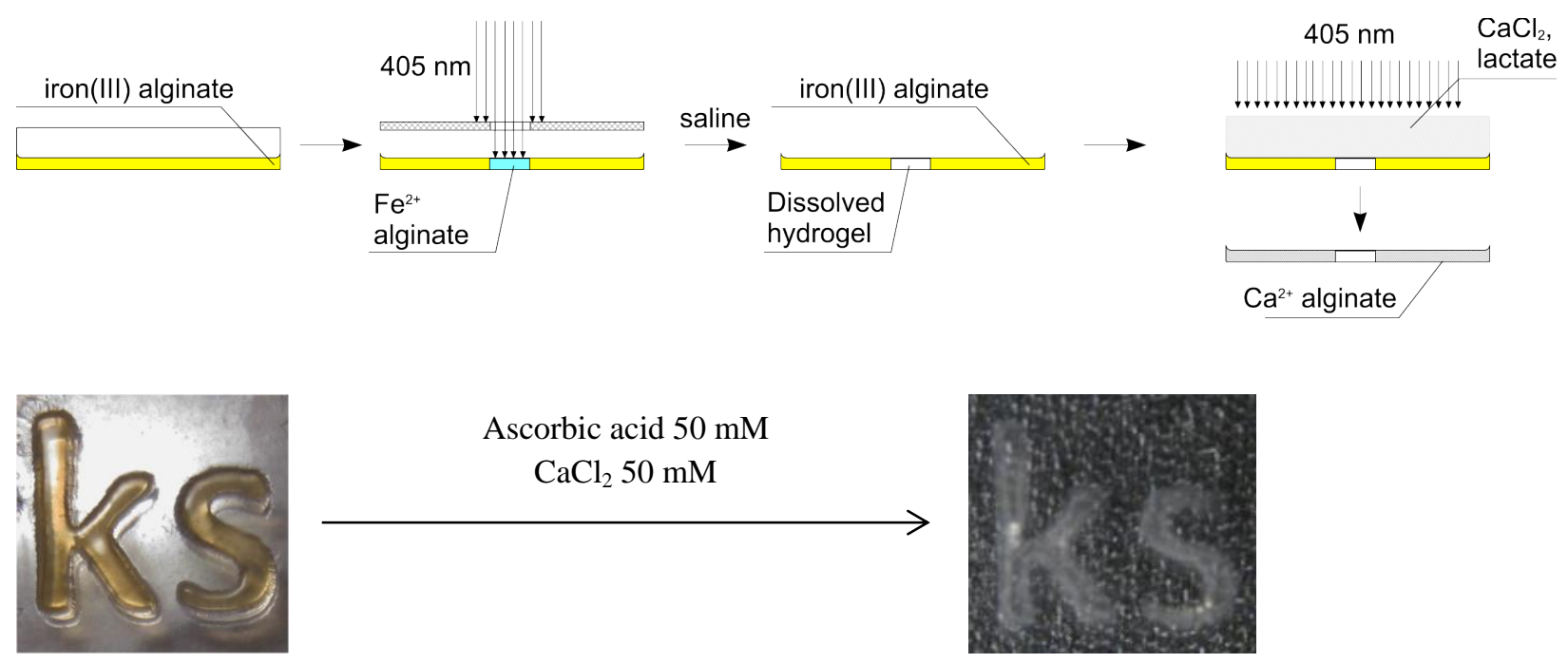
Figure 8. Above: Preparation of patterned calcium cross-linked alginate hydrogel (D) by Photochemical patterning of iron(III) cross-linked alginate hydrogel, dissolution of the exposed areas, and chemical or photochemical reductive cation exchange. Below: Images of patterned iron(III) cross-linked hydrogel (left) and calcium-cross-linked hydrogel obtained from it.

After 6 min of the irradiation the resultant hydrogel was treated with $0.9 \%$ saline solution which dissolved the hydrogel in all exposed areas except ones shielded by printed letters (Fig. 8, bottom left). The obtained letter-patterned iron(III)-cross-linked hydrogels was immersed into $50 \mathrm{mM}$ calcium chloride and $50 \mathrm{mM}$ lactate solution, and irradiated with the same $405 \mathrm{~nm}$ light source. This photochemical reduction of iron(III) cations accompanied by the exchange of the resultant iron(II) cation produced letter-shaped colorless and transparent calcium cross-linked alginate hydrogels (Fig 8, bottom right). These results clearly demonstrate that iron(III) cross-linked alginate hydrogel can serve as a positive photoresist intermediate for subsequent preparation of patterned calcium alginate hydrogel. We also attempted an alternative approach in which the same iron(III) cross-linked hydrogels serves as a negative photoresist using the same irradiation setup for the hydrogel immersed into a solution calcium chloride in order to produce selective photochemical reductive cation exchange only in the exposed areas followed by reductive dissolution of the dark areas. However, under these conditions calcium cations diffuse into the dark areas making the reductive dissolution of these regions impossible.

\section{Conclusions}

We have demonstrated that In the presence of $\mathrm{Ca}^{2+}$ salts, chemical or photochemical reduction of iron(III) cations in iron(III) cross-linked alginate hydrogels results in replacement of the resultant iron(II) cations producing calcium cross-linked alginate hydrogel. Photochemical reduction under these conditions produces homogeneous hydrogel where $\mathrm{Ca}^{2+}$ cations bind polyguluronate domains in the "egg-box" 
fashion. The process of reductive cation exchange is useful for fabrication of patterned calcium alginate hydrogels using photochemical patterning of iron(III) cross-linked hydrogel containing sodium lactate at the first stage, and the photochemical reductive cation exchange of iron cations to calcium in the presence of lactic acid.

\section{Acknowledgements}

This work was supported by National Science Foundation grant CHE 1150768.

\section{References}

Alsberg, E., Anderson, K. W., Albeiruti, A., Franceschi, R. T., \& Mooney, D. J. (2001). Cell-interactive alginate hydrogels for bone tissue engineering. Journal of Dental Research, 80(11), 2025-2029.

Bhoi, A. K., Das, S. K., Majhi, D., Sahu, P. K., Nijamudheen, A., Anoop, N., Rahaman, A., \& Sarkar, M. (2014). Analyte Interactions with a New Ditopic Dansylamide-Nitrobenzoxadiazole Dyad: A Combined Photophysical, NMR, and Theoretical (DFT) Study. J. Phys. Chem. B, 118(33), 9926-9937.

Boateng, J. S., Matthews, K. H., Stevens, H. N. E., \& Eccleston, G. M. (2008). Wound healing dressings and drug delivery systems: A review. Journal of Pharmaceutical Sciences, 97(8), 2892-2923.

Bracher, P. J., Gupta, M., \& Whitesides, G. M. (2009). Shaped Films of Ionotropic Hydrogels Fabricated Using Templates of Patterned Paper. Advanced Materials, 21(4), 445-450.

Bruchet, M., Mendelson, N., \& Melman, A. (2013). Photochemical Patterning of Ionically Cross-Linked Hydrogels. Processes, 1(2), 153-166. 
Chueh, B. H., Zheng, Y., Torisawa, Y. S., Hsiao, A. Y., Ge, C. X., Hsiong, S., Huebsch, N., Franceschi, R., Mooney, D. J., \& Takayama, S. (2010). Patterning alginate hydrogels using light-directed release of caged calcium in a microfluidic device. Biomedical Microdevices, 12(1), 145-151.

Grant, G. T., Morris, E. R., Rees, D. A., Smith, P. J. C., \& Thom, D. (1973). Biological interactions between polysaccharides and divalent cations. Egg-box model. FEBS (Fed. Eur. Biochem. Soc.) Lett., 32(1), 195-198.

Grellier, M., Granja, P. L., Fricain, J. C., Bidarra, S. J., Renard, M., Bareille, R., Bourget, C., Amedee, J., $\&$ Barbosa, M. A. (2009). The effect of the co-immobilization of human osteoprogenitors and endothelial cells within alginate microspheres on mineralization in a bone defect. Biomaterials, 30(19), 3271-3278.

Griffin, D. R., \& Kasko, A. M. (2012). Photodegradable Macromers and Hydrogels for Live Cell Encapsulation and Release. Journal of the American Chemical Society, 134(31), 13103-13107.

Jin, Z., Guven, G., Bocharova, V., Halamek, J., Tokarev, I., Minko, S., Melman, A., Mandler, D., \& Katz, E. (2011). Electrochemically Controlled Drug-Mimicking Protein Release from Iron-Alginate Thin-Films Associated with an Electrode. ACS Applied Materials \& Interfaces, 4(1), 466-475.

Kim, Y. J., Feng, X. D., \& Lippard, S. J. (2007). Synthesis, structure, and properties of a mixed-valent triiron complex of tetramethyl reductic acid, an ascorbic acid analogue, and its relationship to a functional non-heme iron oxidation catalyst system. Inorganic Chemistry, 46(15), 6099-6107.

Kisiday, J., Jin, M., Kurz, B., Hung, H., Semino, C., Zhang, S., \& Grodzinsky, A. J. (2002). Selfassembling peptide hydrogel fosters chondrocyte extracellular matrix production and cell division: Implications for cartilage tissue repair. Proceedings of the National Academy of Sciences of the United States of America, 99(15), 9996-10001.

Kuo, C. K., \& Ma, P. X. (2001). Ionically crosslinked alginate hydrogels as scaffolds for tissue engineering: Part 1. Structure, gelation rate and mechanical properties. Biomaterials, 22(6), 511-521. 
Lee, K. Y., \& Mooney, D. J. (2011). Alginate: Properties and biomedical applications. Progress in Polymer Science, 37(1), 106-126.

Li, L. B., Fang, Y. P., Vreeker, R., \& Appelqvist, I. (2007). Reexamining the egg-box model in calciumalginate gels with X-ray diffraction. Biomacromolecules, 8(2), 464-468.

Machida-Sano, I., Matsuda, Y., \& Namiki, H. (2009). In vitro adhesion of human dermal fibroblasts on iron cross-linked alginate films. Biomedical Materials, 4(2), 1-8.

Machida-Sano, I., Ogawa, S., Ueda, H., Kimura, Y., Satoh, N., \& Namiki, H. (2012). Effects of Composition of Iron-Cross-Linked Alginate Hydrogels for Cultivation of Human Dermal Fibroblasts. International Journal of Biomaterials, 2012, 8.

Morris, E. R., Rees, D. A., Thom, D., \& Boyd, J. (1978). Chiroptical and stoichiometric evidence of a specific, primary dimerisation process in alginate gelation. Carbohydrate Research, 66(1), 145-154.

Narayanan, R. P., Melman, G., Letourneau, N. J., Mendelson, N. L., \& Melman, A. (2012). Photodegradable Iron(III) Cross-Linked Alginate Gels. Biomacromolecules, 13(8), 2465-2471.

Orive, G., Carcaboso, A. M., Hernandez, R. M., Gascon, A. R., \& Pedraz, J. L. (2005). Biocompatibility evaluation of different alginates and alginate-based microcapsules. Biomacromolecules, 6(2), 927-931.

Ray Sarkar, B. C., \& Chauhan, U. P. S. (1967). A new method for determining micro quantities of calcium in biological materials. Analytical Biochemistry, 20(1), 155-166.

Re'em, T., Tsur-Gang, O., \& Cohen, S. (2010). The effect of immobilized RGD peptide in macroporous alginate scaffolds on TGF beta 1-induced chondrogenesis of human mesenchymal stem cells. Biomaterials, 31(26), 6746-6755.

Ruvinov, E., Leor, J., \& Cohen, S. (2011). The promotion of myocardial repair by the sequential delivery of IGF-1 and HGF from an injectable alginate biomaterial in a model of acute myocardial infarction. Biomaterials, 32(2), 565-578. 
Shapiro, L., \& Cohen, S. (1997). Novel alginate sponges for cell culture and transplantation. Biomaterials, 18(8), 583-590.

Sikorski, P., Mo, F., Skjak-Braek, G., \& Stokke, B. T. (2007). Evidence for egg-box-compatible interactions in calcium-alginate gels from fiber X-ray diffraction. Biomacromolecules, 8(7), 2098-2103.

Tan, W. H., \& Takeuchi, S. (2007). Monodisperse alginate hydrogel microbeads for cell encapsulation. Advanced Materials, 19(18), 2696-2701.

Tang, M., Chen, W., Weir, M. D., Thein-Han, W., \& Xu, H. H. K. (2012). Human embryonic stem cell encapsulation in alginate microbeads in macroporous calcium phosphate cement for bone tissue engineering. Acta Biomaterialia, 8(9), 3436-3445.

Theil, E. C., \& Goss, D. J. (2009). Living. with Iron (and Oxygen): Questions and Answers about Iron Homeostasis. Chemical Reviews, 109(10), 4568-4579.

Valko, M., Rhodes, C. J., Moncol, J., Izakovic, M., \& Mazur, M. (2006). Free radicals, metals and antioxidants in oxidative stress-induced cancer. Chemico-Biological Interactions, 160(1), 1-40.

Wang, C. C., Yang, K. C., Lin, K. H., Liu, Y. L., Liu, H. C., \& Lin, F. H. (2012). Cartilage regeneration in SCID mice using a highly organized three-dimensional alginate scaffold. Biomaterials, 33(1), 120-127.

Yu, J. S., Du, K. T., Fang, Q. Z., Gu, Y. P., Mihardja, S. S., Sievers, R. E., Wu, J. C., \& Lee, R. J. (2010). The use of human mesenchymal stem cells encapsulated in RGD modified alginate microspheres in the repair of myocardial infarction in the rat. Biomaterials, 31(27), 7012-7020. 